\title{
"Empowering services based software in the Digital single Market to foster an ecosystem of trusted, interoperable and legally compliant cloud services"
}

\author{
Juncal Alonso Ibarra ${ }^{1}$, Leire Orue-Echevarria ${ }^{1}$, Marisa Escalante ${ }^{1}$, Gorka Benguria ${ }^{1}$ \\ ${ }^{I}$ Tecnalia, Parque Científico y Tecnológico de Bizkaia, C/ Geldo, Edificio 700, Derio, Spain \\ \{juncal.alonso, leire.orue-echevarria,marisa.escalante,gorka.benguria\}@tecnalia.com
}

\begin{abstract}
Keywords: $\quad$ SLA, QoS, Cloud Services, Cloud service Broker, Digital Single Market, Services aggregation, Cloud service intermediator.

Abstract: The software industry has evolved from software on the shelf based applications deployed in dedicated servers, to Software as a service based components running on public or private Clouds and now to Cloud Service Brokers. So, Cloud service brokerages have emerged as digital intermediaries in the information technology (IT) services market (Shang 2013), creating value for cloud computing clients and vendors alike. This paper presents an approach to foster next generation cloud service brokers through an ecosystem of trusted, interoperable and legally compliant cloud services through an added value Cloud Services intermediator. This ecosystem will offer, create, consume and assess trusted, interoperable, and standard Cloud Services, where to (semi-)automatically deploy the next generation service based software applications.
\end{abstract}

\section{INTRODUCTION}

The transformation from product to a service based economy means that companies need to become software service providers as well as consumers. In this context, cloud enables greater business agility by making IT infrastructure more flexible.

Cloud services have already caused a major transformation in technologies and a paradigm shift in business operations focus that has affected everything from ERP to datacenter planning (Markets 2014). Next phases in the Cloud Services evolution include the incorporation of many supplier/customer relationships, and their correlative data exchange and transactions.

The software industry has evolved from software on the shelf based applications deployed in dedicated servers, to Software as a service based components running on public or private Clouds and now to Cloud Service Brokers (CSB). The fundamental CSB functions are (Markets 2014) to aggregate, simplify, secure, and integrate data, communications, and commerce between vendors.
These functions are often referred to as Cloud Intermediation services and include both technical and business process mediation on a cloud-to-cloud basis.

Cloud service brokerages have emerged as digital intermediaries in the information technology (IT) services market, creating value for cloud computing clients and vendors alike (Shang 2013).

According to Gartner Inc (Gartner 2016) and Oracle (Oracle 2014), a cloud services brokerage offers three capabilities:

- Cloud Service Intermediation: An intermediation broker provides value added services on top of existing cloud platforms, such as identity or access management capabilities.

- Aggregation: An aggregation broker provides the "glue" to bring together multiple services and ensure the interoperability and security of data between systems.

- Cloud Service Arbitrage: A cloud service arbitrage provides flexibility and 
"opportunistic choices" by offering multiple similar services to select from.

The next generation of Cloud Services Brokers need to go one step beyond. Aggregation of Cloud Services is not enough as Cloud Services users are experiencing new challenges such as :

- Vendor lock-in: once a company uses a service offered by a cloud provider or deploys an application on a certain cloud provider, it is very likely that the company will be greatly dependent on the platform or services of a cloud provider (e.g. while using their virtualized infrastructure), making it harder to port the application to another cloud provider or use another service offered by another provider.

- Lack of compliance of legislation: the use of cross-border services is currently limited because most of existing cloud services do not comply fully with EU28 legislation and regulations. EU harmonization of legislation in, i.e. data protection, is a recurring barrier. However, cloud computing will only be uptaken when cloud services comply with or aware of EU28 legislation.

- Interoperability: data portability and seamless use of applications that can interoperate with each other are key challenges for cloud consumers. Interoperability among cloud service providers is also an unsolved challenge, especially when using cross-border cloud services.

- SLA assessment: when aggregating cloud services from different cloud providers, SLAs must also be consequently aggregated. However, currenly, SLAs, SLOs and SLA metrics are not standardized making this SLA composition, assessment and monitoring very difficult. Several initiatives in this direction of SLA metrics and SLOs have been launched and are currently ongoing.(SLALOM,2015) (Time.lex, 2015).

This paper presents an approach to foster an ecosystem of trusted, interoperable and legally compliant cloud services through an added value Cloud Services intermediator. This ecosystem will offer, create, consume and assess trusted, interoperable, and standard Cloud Services, where to (semi-)automatically deploy the next generation service based software applications. The paper is organized as follows. First, an introduction to the cloud services ecosystems and their situation is provided. Next the existing solutions in the area are presented. Then the proposed solution is explained in detail, including an overview of the approach as well as the description of the modules composing the solution. The paper finishes with a set of conclusions and next steps to follow.

\section{CLOUD SERVICES ECOSYSTEMS}

Working with many cloud service providers means managing multiple relationships. Most enterprises are already negotiating on a one-to-onebasis multiple contracts with multiple cloud service providers. Multiple contracts mean multiple communication channels, payments, multiple passwords, multiple data streams, multiple interfaces and multiple providers to check up on. That derives on questions about how to make those services work together, how to interact with all of them or how to unify all the efforts so maximum effectiveness and efficiency can be obtained. This is when a Cloud Service Broker (CSB) comes into play. As defined by Plummer (Plummer 2012) a cloud services brokerage is a third-party software that adds value to cloud services on behalf of cloud service consumers. Their goal is to make the service more specific to a company, or to integrate or aggregate added value services, to enhance their non-functional properties such as security, or to do anything which adds a significant layer of value (i.e. capabilities) to the original cloud services being offered. Consumers can leverage solutions offered by CSBs that allow organizations to focus on other business needs instead. A viable CSB provider can make it less expensive, less complex, less risky (also in legal terms), interoperable and more effective for companies to discover, aggregate, consume and extend cloud services, particularly when they span multiple, diverse cloud services providers in different EU Member States.

Some of the key vendors currently occupying the Cloud service brokerage market are Accenture, HP, NEC Corporation, Jamcracker, Gravitant, ComputeNext, Cloud Sherpas, Arrow Electronics, and Capgemini. However, none of these are currently compliant with EU28 legislation nor contain certified services or foster interoperability or 
avoid vendor lock-in. According to Markets and Markets (Markets, 2014), the global Cloud Service Brokerage Market is expected to grow from \$5.24 Billion in 2015 to $\$ 19.16$ Billion by 2020 , at a Compound Annual Growth Rate (CAGR) of 29.6\% from 2015 to 2020 .

\subsection{Current situation and challenges}

With the evolution of cloud computing towards XAAS (Anything as a service), today's organisations have on their hand a plethora of alternatives to fulfil their information technology's needs. For example, while developing or migrating a web site, an organisation can decide to build it in a dedicated internal computer, build it as an instance in a shared internal computer, build it in a dedicated external computer, or even build it as an instance in a shared external. The decision on using one, another, or several approaches simultaneously is driven by certain evaluation criteria (e.g. profitability, reliability, performance, and security, legal or even ecological aspects).

"It is not the strongest of the species that survives, nor the most intelligent, but the one most responsive to change." L.C. Megginson

This quote, often assigned to Charles Darwin, has been frequently used in other domains, Economics, Education, of course Information Technologies (IT). Changes in IT-systems are initiated starting from user requests, monitoring activities or modernization initiatives.

As stated by several Cloud stakeholders, such as Forbes, Cloud enables greater business agility by making IT infrastructure more flexible. It provides software vendors the needed means to enhance their relationship with their customers.. Whether an organization runs a company's applications on data centre hardware, or on a private or public cloud, it still needs to align itself with the business' needs, rather than forcing the business to align itself with IT's. Complex software applications need context aware computing resources ready to react to a changeable environment.

Existing cloud services shall be made available dynamically, broadly and cross border, so that software providers can re-use and combine cloud services, assembling a dynamic and re-configurable network of interoperable, legal compliant, quality assessed (against SLAs) single and composite cloud services.

With so much activity implementing front-end and back-end applications in public and private clouds, complexity has grown at every level (business, application, transaction and regulatory) (Oracle 2014).

Business transactions span multiple clouds and therefore it is necessary to integrate clouds at the transaction level.

To generate meaningful results, Oracle envisions that enterprises need to address key challenges in the next years (Oracle 2014):

1. Governance: Ensuring that services deployed in the cloud are protected is critical. Sharing can create leaks that cannot be tolerated. Fostering strong governance programs in place will protect enterprises and their data.

2. Risk tolerance. Every enterprise should assess their tolerance for pitfalls such as lost data and application outages. As Information as a Service and Integration as a Service evolve, enterprises will see risks reduced.

3.Regulations. Lobbying for regulations and standards are predicted to be a key step to ensure cloud integration.

4. Cross border interoperability: The resulting service intermediator shall support intelligent discovery, context-aware service management and fluid service integration, assuring data portability in such a federated ecosystem, while guaranteeing proper identity propagation with service-specific granularity level of information.

5. Matching customer requirements with cloud service specifications: customers in any EU country should be provided with a guarantee of security, legislation awareness and other non-functional requirements when using any cross-border service within heterogeneous environment. This implies that the selected service offerings must match with all functional and non-functional requirements coming from the customers.

6. Legislation compliance, defining means of assuring service compliance with legislation of EU countries: a service is legislation aware when the services are constrained by legal requirements, such as data privacy, data protection, data security and data location. Moreover, a big challenge in this concern is to develop the methods and interfaces for securing legislation compliance and easy legislation change propagation through the cross-bordered and composite services in a legislation heterogeneous environment.

7. Cloud service SLA assessment and monitoring: monitor and control the diverse properties of utilized services, composite or standalone, at real-time, while also being able to provide all the critical information for the appropriate 
reactions when necessary, especially when SLA conditions are not fulfilled (e.g. elasticity, data localisation).

8. Seamless change of provider: enable to seamlessly change the service provider including all services, dependencies and associated data to avoid vendor lock-in and to be able to quickly react in situations like bankruptcy of the cloud provider or any other cases which causes outage of the service.

\subsection{Existing solutions}

According to MarketsandMarkets (Markets,2014), the Cloud Brokerage Enablement market will grow from $\$ 225.42$ million in 2013 to $\$ 2.03$ billion by 2018, at a CAGR of 55.3 percent. Cloud Brokerage Enablers are software platforms that enable such services. The overall, global Cloud Brokerage market will grow from $\$ 1.57$ billion in 2013 to $\$ 10.5$ billion by 2018. This represents a CAGR of 46.2 percent from 2013 to 2018.

Several private Cloud Service Brokers have been active known players in this market (Gartner, 2012) (Jamcracker, Gravitant, Computenext, HP, Dell, Nephos Technologies, NEC, Cloud Sherpas and Capgemini) .Competition may also intensify with an influx of new players, like CompatibleOne, Gravitant or CloudSelect.

All of these are mostly focused on providing service brokerage of Cloud Service Providers, mainly VM's and virtualized resources, but not of other services offered (e.g. Data Processing as a Service) or SaaS applications which are certified and legally compliant.

Currently, according to the European Commission and their report of the Digital Single Market (European Commission, 2015) only 4\% of the services offered are cross-border, while $42 \%$ are national and 54\% are US-based.

The proposed solution aims to increase the provisioning and consumption of legally compliant, cross - border services.

\section{ADDED VALUE CLOUD SERVICE INTERMEDIATOR}

\subsection{Presented approach}

The proposed cloud service metaintermediator is an intermediate layer that will ease the discovery and adoption of Cloud services, single services discovered from a cloud service provider or composite ones coming from running $\mathrm{CSB}$, in which to deploy service based software and the creation of a sustainable ecosystem of certified, accredited and trustworthy applications. Some modules or functionalities that will include the service metaintermediator are: service discovery, service composition, legislation awareness, interoperability, identity propagation, monitoring, billing, SLA assessment, service registry, legislation databases, among others.

\subsection{Proposed solution}

In the following section, an overview of the modules and functionalities of the proposed solution is presented:

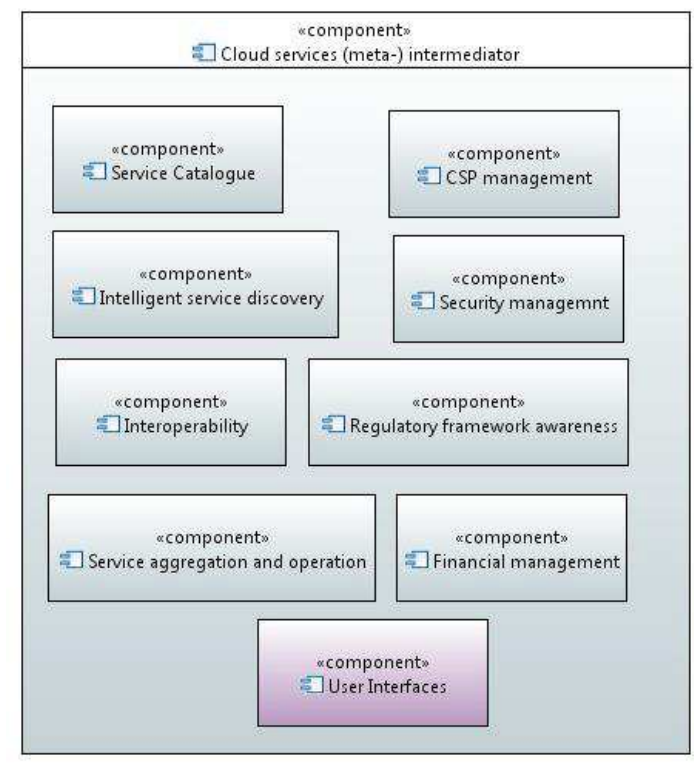

Figure 1. Cloud service (meta-) intermediator architecture overview.

- Service catalogue: This component will include the required functional components for cataloguing the services to be included in the in the service intermediator:

- Service registry: The service registry will follow a declarative service configuration template and will include information about CSLA of the service, service life-cycle status, service 
consumers or service category among others.

- Service registry governance \& follow up: These modules are in charge of governing the service registration process, service deletion, etc.

- Intelligent service discovery: Automatic identification of service and service benchmarking based on the needs and requirements with respect to different criteria (non-functional properties, legislation compliance, prize, etc.) stablished by the user.

- Service aggregation and operation: The framework will provide the required modules to provide composite (aggregated) services formed of unitary services from the same or different Cloud Service Providers able to meet users' functional and non-functional requirements.

- CSP management: This module is in charge of controlling the relationships with the Cloud Service Provider including the management of SLA of their provided Cloud Services. The submodules envisioned are:

- CSLA assessment of the service in operation, i.e., the assessment of whether the non-functional properties are within both the thresholds specified by the legislation as well as the thresholds specified in the contract. Properties such as performance, availability and resilience, etc. will be monitored and assessed.

- CSLA monitoring: This module also includes the monitoring of the CSLA. The continuous real-time monitoring of the non-functional properties specified in the CSLA will be done through a set of components one for each property. These modules are in charge of measuring the values of the metrics of the SLOs in the contract, be them related to QoS or QoSec. The modules work in a non-intrusive way in form of testing the cloud services as an independent party.

- CSLA Definition: this module is in charge of the transformation of National
CSP offers into standard CSLAs (included standard metrics).

- Security Management module which includes different sub-modules for assessing the secure operation of the framework and the different services provided. The sub-modules to be included are:

- Access and rights management: These modules will uniquely identify and authenticate the users ecosystem. The module will also be in charge of the internal identification and authentication of the modules in operation.

- Identity Management: This module is in charge of managing the identity propagation in the (meta) service intermediator following a distributed architecture approach.

- Anonymization: This sub-module will be in charge of anonymizing the data of the service users when needed.

- Regulatory framework awareness: This module will contain European and Member states legislations in a structured data format based in a standardized data model. The relevant provisions in each legislation will be linked to non-functional service level metrics and their acceptable thresholds, so the legislation compliance of the services can be checked against these predefined metrics. Once established the service level metrics and thresholds related to legislative provisions, this module will evaluate even before service operation starts if the service is compliant with European and National regulations. These module will include at least the following submodules to perform the described legislation awareness process:

- Standards and EU and MMSS legislation database

- Regulation compliance assessment

- Standards and EU and MMSS sources management

- Regulations change history

- Regulations compliance monitoring

- Interoperability: This module is responsible of being the data transfer mechanism between different cloud services. This module will make it possible for services using nonstandard and proprietary data formats and protocols to communicate with the bus through specific connectors for each type of 
service and as a result it will execute the migration process necessary for seamlessly data portability. This module will also include the connector management for the different Cloud Service Providers, so they can offer their services through the intermediator.

- Financial management: This module includes the standard functions of a cloud service broker such as accounting, metering and billing of service instances in operation.

- Service intermediator user interfaces: The framework will include the user interfaces for the different users. Here, we distinguish between users that are using different services of the intermediator, administration users and CSPs users of the framework The different user interfaces envisaged as user console, administration console and CSP console will include sub-modules such as, dashboards, notification and alert managements, as well as others personalized for each type of user.

\section{CONCLUSIONS}

As stated by the European Commission, the internet and digital technologies are transforming our world. But existing barriers online mean citizens miss out on goods and services, internet companies and startups have their horizons limited, and businesses and governments cannot fully benefit from digital tools.

It is time to make the EU's single market fit for the digital age - tearing down regulatory walls and moving from 28 national markets to a single one. This could contribute $€ 415$ billion per year to our economy and create hundreds of thousands of new jobs.

Cloud Service Brokers are much like any other type of broker in this case, acting on behalf of customers to aggregate, integrate and customize cloud offerings by multitudes of providers.

This paper proposes a solution to implement the next generation of Cloud Service Brokers, providing an intermediate layer that will ease the federation of Cloud Service Brokers, as well as the discovery and adoption of Cloud services on which to deploy service based software and the creation of a sustainable ecosystem of certified, accredited and trustworthy applications.

Next steps include the actual implementation of the design presented in this paper and the validation in real life industrial and/or public administration scenarios.

\section{ACKNOWLEDGEMENTS}

This work has been partially funded by the European project Cloud for Europe (Seventh Framework Programme for research, technological development and demonstration under grant agreement no 610650).

\section{REFERENCES}

SLALOM, (2015). http://slalom-project.eu/

Time.lex, Sparks (2015), "Standards terms and performance criteria in service level agreements for cloud computing services"https://ec.europa.eu/digitalagenda/en/news/study-report-standards-terms-andperformances-criteria-service-level-agreements-cloudcomputing

Markets (2014), "Cloud Services Brokerage: Technology and Market Assessment 2014 - 2019"

https://www.reportbuyer.com/product/2589608/cloudservices-brokerage-technology-and-marketassessment-2014-2019.html

Oracle (2014), "Making waves, Cloud brokerage and integration".

http://www.oracle.com/us/solutions/cloud/managed-cloudservices/makingwaves-final-2162934.pdf

Gartner. (2012). "Predicts 2013: Cloud Services Brokerage”, Gartner Research Report, ID Number:

G00239877, December 18, 2012

https:/www.gartner.com/doc/2985118?srcId=1$2819006590 \& p c p=i t g$

European Commission (2015) "A digital single market for Europe”. http://ec.europa.eu/priorities/digital-singlemarket/docs/dsm-1-year en.pdf

Shang et al. (2013) "Analyzing the impact of brokered services on the Cloud Computing Market", http://docplayer.net/2008961-Analyzing-the-impactof-brokered-services-on-the-cloud-computing$\underline{\text { market.html }}$

Gartner

http://www.gartner.com/technology/home.jsp

Daryl Plummer (2012) "Cloud Services Brokerage: A Must-Have for Most Organizations" http://www.forbes.com/sites/gartnergroup/2012/03/22/ cloud-services-brokerage-a-must-have-for-mostorganizations/\#2715e4857a0b12e1dd5252aa 